\title{
3D pulmospheres serve as a personalized and predictive multicellular model for assessment of antifibrotic drugs
}

\author{
Ranu Surolia, ${ }^{1}$ Fu Jun Li, ${ }^{1}$ Zheng Wang, ${ }^{1}$ Huashi Li, ${ }^{1}$ Gang Liu, ${ }^{1}$ Yong Zhou, ${ }^{1}$ Tracy Luckhardt, ${ }^{1}$ \\ Sejong Bae, ${ }^{2}$ Rui-ming Liu, ${ }^{1}$ Sunad Rangarajan, ${ }^{1}$ Joao de Andrade, ${ }^{1,3}$ Victor J. Thannickal,, ${ }^{1,3}$ \\ and Veena B. Antony ${ }^{1}$ \\ 'Division of Pulmonary, Allergy, and Critical Care Medicine and 'Division of Preventative Medicine, Department of Medicine, \\ University of Alabama at Birmingham, Birmingham, Alabama, USA. ${ }^{3}$ Birmingham VA Medical Center, \\ Birmingham, Alabama, USA.
}

\begin{abstract}
Idiopathic pulmonary fibrosis (IPF) is a fatal progressive fibrotic lung disease characterized by the presence of invasive myofibroblasts in the lung. Currently, there are only two FDA-approved drugs (pirfenidone and nintedanib) for the treatment of IPF. There are no defined criteria to guide specific drug therapy. New methodologies are needed not only to predict personalized drug therapy, but also to screen novel molecules that are on the horizon for treatment of IPF. We have developed a model system that exploits the invasive phenotype of IPF lung tissue. This ex vivo 3D model uses lung tissue from patients to develop pulmospheres. Pulmospheres are 3D spheroids composed of cells derived exclusively from primary lung biopsies and inclusive of lung cell types reflective of those in situ, in the patient. We tested the pulmospheres of $\mathbf{2 0}$ subjects with IPF and 9 control subjects to evaluate the responsiveness of individual patients to antifibrotic drugs. Clinical parameters and outcomes were also followed in the same patients. Our results suggest that pulmospheres simulate the microenvironment in the lung and serve as a personalized and predictive model for assessing responsiveness to antifibrotic drugs in patients with IPF.
\end{abstract}

Conflict of interest: The authors have declared that no conflict of interest exists.

Submitted: October 21, 2016 Accepted: December 12, 2016 Published: January 26, 2017

Reference information: JCI Insight. 2017;2(2):e91377. doi:10.1172/jici.insight.91377.

\section{Introduction}

Idiopathic pulmonary fibrosis (IPF) is a chronic progressive fibrosing lung disease of unknown etiology characterized by a histopathological or radiological pattern of usual interstitial pneumonia. It has an unpredictable clinical course, with a median survival of only 3 years following diagnosis (1). Treatment options are limited, and the novel antifibrotic agents nintedanib and pirfenidone slow down the rate of decline in forced vital capacity (FVC) and improve quality and length of life $(2,3)$. More effective therapies are urgently needed, but one of the major limitations in the development of drug therapies in IPF is the lack of reliable preclinical models of the disease. Current animal models, primarily those in rodents, have been disappointing (4), and many promising drugs have failed to demonstrate efficacy in human randomized clinical trials. An ideal preclinical model will need to recapitulate the multitude of factors responsible for the disease phenotype or endotype $(1,5,6)$. Furthermore, it would more accurately predict drug responsiveness, while providing new insights into the pathobiology of the disease.

Fibrosis is a multicellular complex disease process that is difficult to recapitulate in ex vivo cell culture systems $(7,8)$. An important advance in cell culture techniques has been the development of $3 \mathrm{D}$ culture systems, which better simulate in vivo biochemical cues and cell-cell interactions. In contrast to 2D cell culture, 3D systems reconstituted from primary lung tissue may better replicate cell-cell communications as well as cell-autonomous and cell nonautonomous contributions $(8,9)$. Another advantage of primary lung cell 3D cultures is that they can be cryopreserved for future analyses (10).

IPF is characterized by the presence of invasive myofibroblasts. Recent studies from our group and others have implicated a critical role of the invasive phenotype of myofibroblasts in disease pathogenesis $(7,11)$. However, this profibrotic cellular phenotype has been primarily studied in $2 \mathrm{D}$ cell systems, and the relevance to in vivo pathobiology is unclear (9). In this study, we explored the utility of 3D primary cell culture systems in creating an ex vivo model of the IPF lung. We specifically sought to test 
the behavior of invasive myofibroblasts and to explore the ability of $3 \mathrm{D}$ primary cell culture systems to predict the in vivo responsiveness to specific antifibrotic drugs.

\section{Results}

We prospectively recruited 20 consecutive subjects who required surgical lung biopsy for the diagnosis of IPF. Lung samples from 9 donors whose lungs were not utilized for lung transplant for nonlung disease reasons served as controls.

Pulmospheres are 3D structures made of multiple types of cells and extracellular matrix. Lung tissues from controls and IPF subjects were processed into pulmospheres (Figure 1A). Pulmospheres were 3D multicellular structures as visualized by phase-contrast microscopy and H\&E staining. Immunofluorescence affirmed that control lung pulmospheres and IPF lung pulmospheres were comprised of various lung cell types. Control pulmospheres were positive for surfactant protein C (SPC, a marker for epithelial type II cells), CD31 (endothelial cells), CD11b (macrophages), and $\alpha$ smooth muscle actin ( $\alpha$-SMA) (vascular smooth muscle cells and myofibroblasts) (Figure 1B). All these various types of cells were embedded in extracellular matrix (ECM) proteins - collagen type I, fibronectin-EDA, and collagen type IV (Figure 1B). The IPF pulmospheres also demonstrated presence of $\alpha$-SMA-positive, SPC-positive, CD31-positive, and CD11b-positive cells (Figure 1C). Immunofluorescence staining demonstrated enhanced staining for ECM proteins, such as collagen type I, fibronectin-EDA, and collagen IV (Figure 1C). These architectural features of lung pulmospheres resembled in vivo conditions in which cells are supported and surrounded by ECM proteins.

Cell phenotypes and protein expression in pulmospheres. The proportion of cells expressing various cell markers was assessed in control and IPF pulmospheres using FACS. Control pulmospheres cells had a significantly lower proportion of CD11b-positive cells compared with IPF pulmospheres $(0.8825 \% \pm 0.35 \%$ vs. $17.72 \%$ $\pm 1.87 \%$, respectively, $P<0.05$ ). There was a significant difference in the proportion of CD31-positive cells between control and IPF pulmospheres $(5.52 \% \pm 0.44 \%$ vs. $35.24 \% \pm 12.54 \%$, respectively, $P=0.05$ ) (Figure 2, A and B). The proportion of SPC-positive cells was increased in IPF pulmospheres compared with control pulmospheres $(38.43 \% \pm 15.65 \%$ vs. $15.75 \% \pm 5.34 \%$, respectively, $P<0.05$ (Figure $2, \mathrm{~A}$ and B).

To evaluate if there were alterations in markers of cellular differentiation, we next evaluated the expression of $\alpha$-SMA and FSP1, comparing cultures of cells obtained from patients with IPF in 2D and 3D culture. The protein analysis of cultured cells in monolayers (2D) used for IPF pulmosphere preparation at passage 0 and IPF pulmospheres (3D) demonstrated upregulated expression of $\alpha$-SMA and FSP1 (patients, $n=3$ ) (Figure 2C). The densitometry analysis revealed a 4 -fold increase in $\alpha$-SMA expression in monolayer (2D) cultures (Figure 2D). FSP1 expression was significantly increased in monolayer culture (2D), when compared with pulmospheres (3D) (Figure 2E). These findings suggest that IPF pulmospheres in 3D culture have significant quantitative and qualitative differences compared with the same cells grown in $2 \mathrm{D}$ culture.

IPF lung pulmospheres are marked by a highly invasive phenotype. Myofibroblasts are a hallmark of fibrotic disorders $(12,13)$. Immunostaining of control and IPF lung pulmospheres with $\alpha$-SMA affirmed the presence of myofibroblasts in IPF pulmospheres. $\alpha$-SMA-positive cells radiated outward from the central core of the IPF lung pulmospheres (Figure 3A). These results were confirmed by FACS analysis (Figure 3B). IPF pulmospheres demonstrated increases in $\alpha$-SMA-positive cells compared with control pulmospheres $(31.33 \% \pm$ $20.5 \%$ vs. $3.7 \% \pm 2.78 \%$, respectively) (Figure $3 \mathrm{C}$ ). We next wanted to evaluate if the increased number of $\alpha$-SMA-positive cells was the cause of the enhanced invasiveness of pulmospheres from subjects with IPF. We tested this possibility by quantitating invasiveness, defined as the zone of invasion percentage (ZOI\%), within each pulmosphere (Figure 3D). We measured the inner core and the total areas of each pulmosphere. The zone of invasion (ZOI) is the area covered by invasive cells and was determined by subtracting the inner core area from the total area of each pulmosphere (Figure $3 \mathrm{E})$. We measured the ZOI\% of control $(n=9)$ and IPF $(n=20)$ pulmospheres. The results demonstrated that the IPF pulmospheres had a higher ZOI\% as compared with control pulmospheres $(158.2 \% \pm 17.88 \%$ vs. $54.99 \% \pm 6.77 \%$, respectively) (Figure $3 \mathrm{~F}$ ). These data demonstrated that IPF lung pulmospheres have a high number of $\alpha$-SMA-positive cells and that enhanced invasiveness was a defining characteristic when compared with control lung pulmospheres.

Modulation of invasiveness by treatment with TGF- $\beta 1$ in a control pulmosphere and nintedanib in a IPF pulmosphere. TGF- $\beta 1$ treatment induces fibroblast differentiation into myofibroblasts (14) and increases their invasiveness. We tested this phenomenon in control lung pulmospheres. Control pulmospheres treated with TGF- $\beta 1$ demonstrated increased invasiveness and had a higher ZOI\% than untreated control lung pulmospheres. Phalloidin-stained and TGF- $\beta 1$-treated control pulmospheres demonstrated prominent invasion of cells away from the inner core 
A
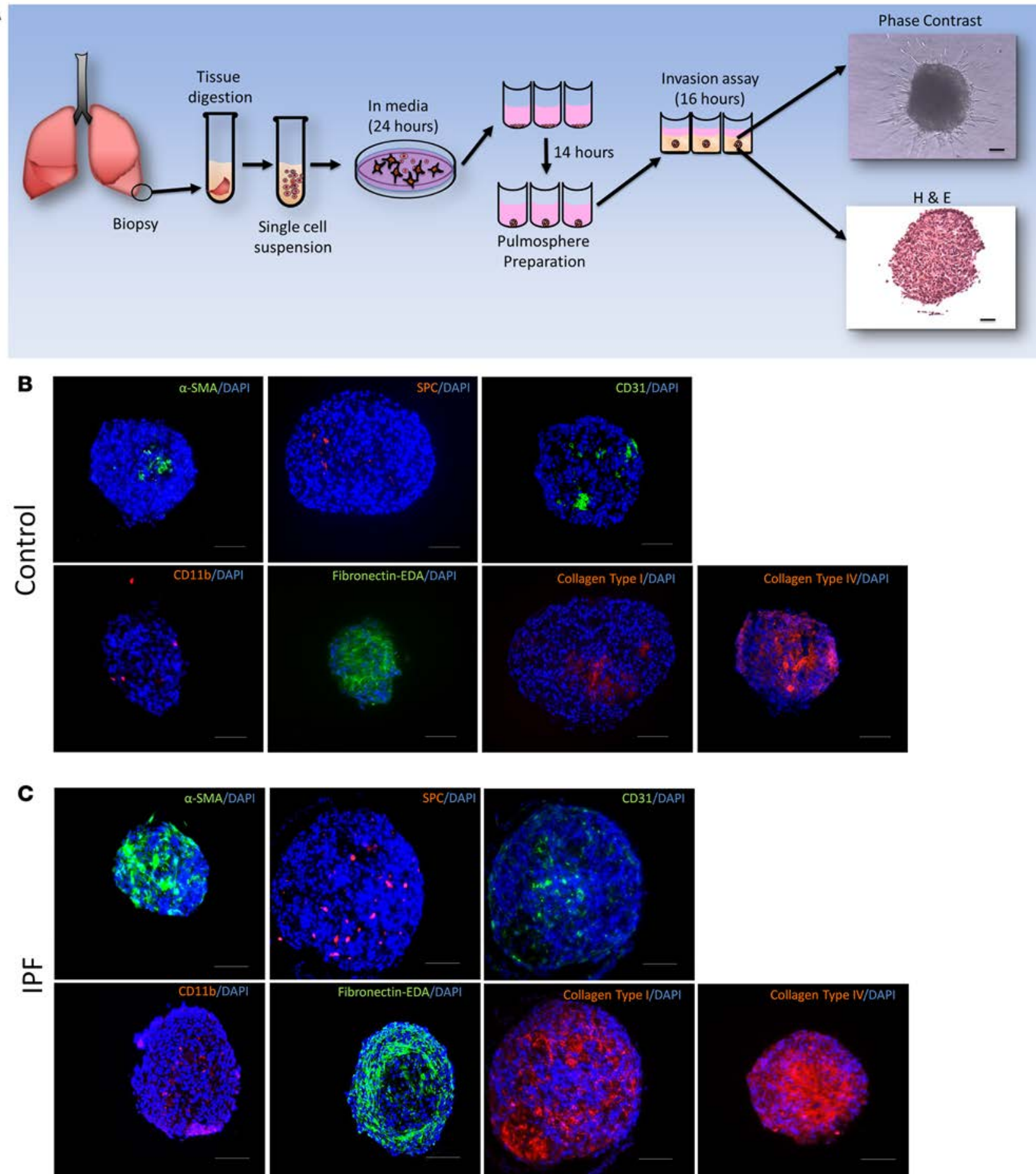

Figure 1. Pulmospheres are 3D structures made of multiple cell types and extracellular matrix. (A) Schematic representation of pulmosphere preparation. Representative phase-contrast microscopic image. H\&E-stained pulmosphere. Scale bar: $250 \mu \mathrm{m}$. (B) Immunofluorescent staining of pulmospheres from IPF patients for $\alpha$-SMA, SPC, CD31, CD11b, fibronectin-EDA, collagen type I, and collagen type IV in paraffin-embedded sections. All immunofluorescent slides were costained with DAPI for nuclei. Five random pulmospheres were sampled from each control subject $(n=9)$. Scale bar: $250 \mu \mathrm{m}$. (C) Immunofluorescent staining of pulmospheres from IPF patients for $\alpha$-SMA, SPC, CD31, CD11b, fibronectin-EDA, collagen type I, and collagen type IV in paraffin-embedded sections. All immunofluorescent slides were costained with DAPI for nuclei. Five random pulmospheres were sampled from each IPF patient $(n=20)$. Scale bar: $250 \mu \mathrm{m}$. 
A
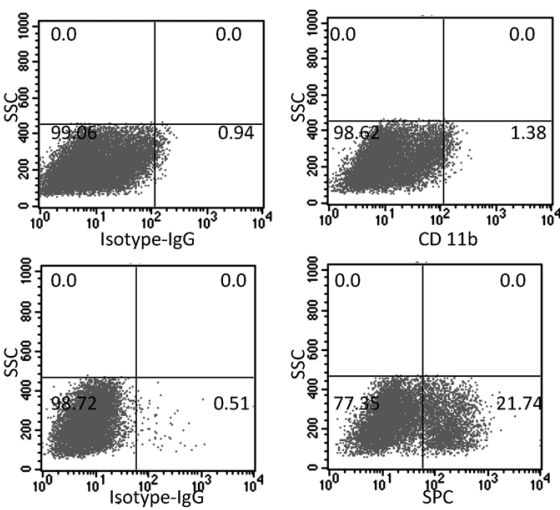

Control

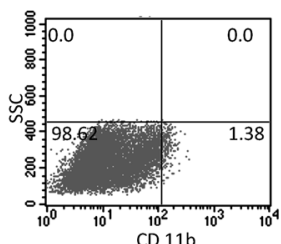

B
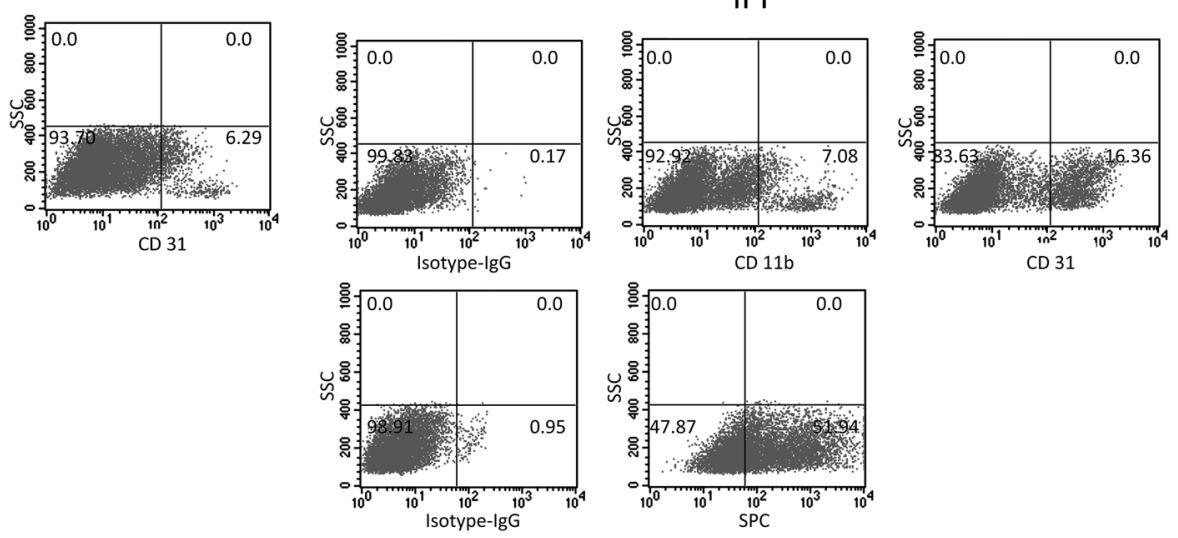

C

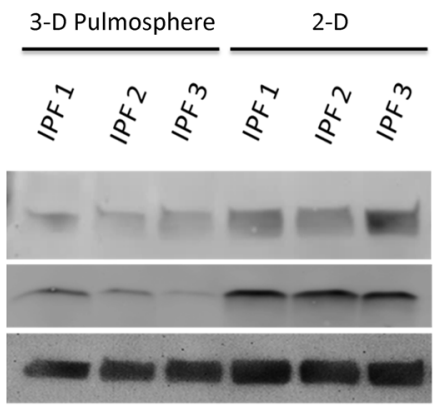

D

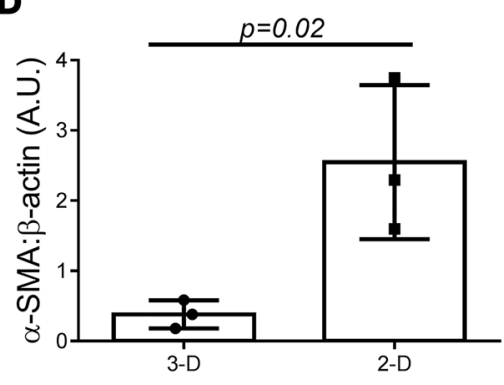

E

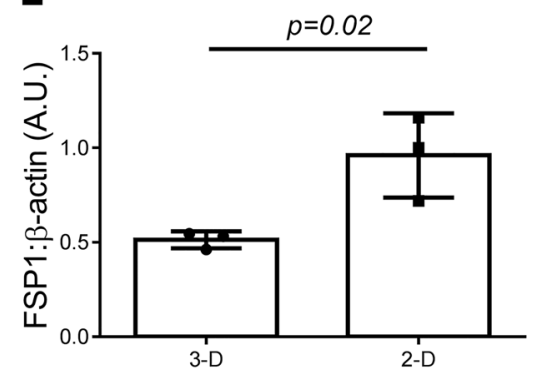

Figure 2. Cell phenotypes and protein expression in pulmospheres. FACS analysis of cells of dissociated pulmospheres from (A) control subjects $(n=9)$ and (B) IPF patients ( $n=20)$ for CD11b and CD31 (top) and SPC (bottom) gated for PE and AlexaFluor 647. PE-conjugated isotype and AlexaFluor 647-conjugated isotypes were used as controls. Seven pulmospheres from each control subject $(n=9)$ and IPF patient $(n=20)$ were used for FACS analysis. SSC, side scatter. (C) The representative Western blot for the expression of $\alpha$-SMA and fibroblast-specific protein 1 (FSP1) in 3D and 2D grown cells from 3 IPF patients. $\beta$-Actin was used as loading control. ( $\mathbf{D}$ and $\mathbf{E}$ ) Quantitative densitometry of Western blots for (D) $\alpha$-SMA and (E) FSP1. $P=0.02$, compared with 3D, unpaired $t$ test. Each dot represents one patient. The Western blotting was repeated independently twice.

of pulmospheres (Figure 4, A and B). Treatment of IPF pulmospheres with nintedanib demonstrated inhibition of invasion and decreased ZOI\% (Figure 4, C and D). These data indicated that exposure to TGF- $\beta 1$ transformed control lung pulmospheres into invasive pulmospheres and that treatment with nintedanib reduced the ZOI, suggesting that invasiveness of lung pulmospheres could be modulated.

Differential invasiveness of pulmospheres from patients with IPF following exposure to nintedanib and pirfenidone. All IPF pulmospheres were prepared and tested for responsiveness to the two FDA-approved antifibrotic drugs, nintedanib $(1 \mu \mathrm{M})$ or pirfenidone $(1 \mathrm{mM})$. The concentrations of nintedanib and pirfenidone were chosen from previous studies performed on primary human lung cells derived from IPF patients (15-17). The responses of all patients to the tested anti-IPF drug were evaluated and represented as fold change in ZOI. Fold change in ZOI was calculated as the ratio in IPF pulmospheres of ZOI\% with treatment to $\mathrm{ZOI} \%$ without treatment (Figure $5 \mathrm{~A}$ ). A ratio less than 1 was defined as inhibition of invasiveness in this in vitro analysis. Conversely, if the ratio was more than 1 , it was defined as an increase in invasiveness (Figure $5 \mathrm{~B})$. Due to patient-to-patient variation in the response, we specified that a patient was responsive to treatment if the median value of fold change in ZOI was less than 1 . There was a decrease in the ZOI in 16 of $20(80 \%)$ pulmospheres treated with nintedanib (exact 95\% confidence interval, $56.3 \%$ to $94.3 \%$ ) (Figure $5 \mathrm{C}$ ), whereas 4 other patient pulmospheres demonstrated an increase in the ZOI (Figure 5C). There was a decrease in the ZOI in 15 of 20 (75\%) pulmospheres treated with pirfenidone (exact $95 \%$ confidence interval, $50.9 \%$ to $91.3 \%$ ) (Figure $5 \mathrm{D}$ ). These data demonstrate a variable invasiveness of lung pulmospheres from IPF subjects and that this invasiveness was altered by treatment with nintedanib or pirfenidone.

Clinical disease progression in patients with IPF correlated with invasiveness of pulmospheres. Following lung biopsy and the formal diagnosis of IPF, 12 of the 20 subjects enrolled in this study were on maintenance 
A Non-IPF
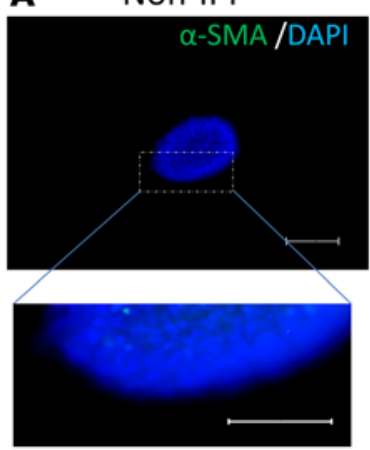

D

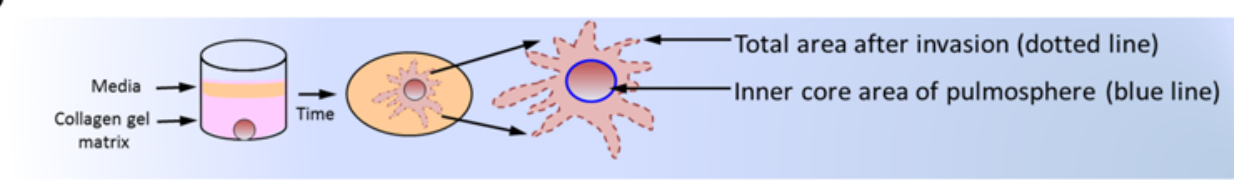

E

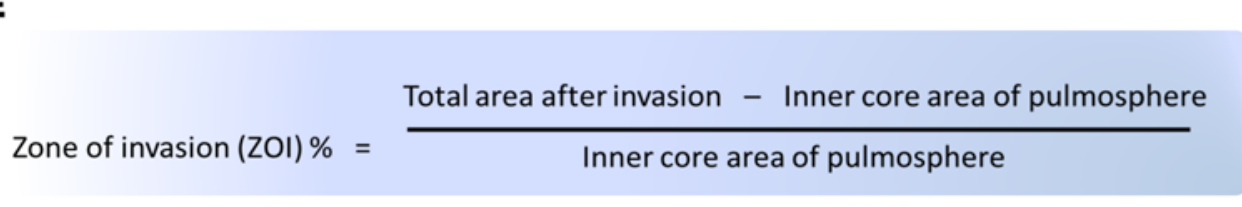

B
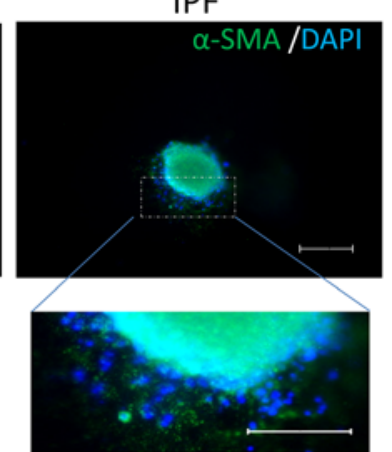
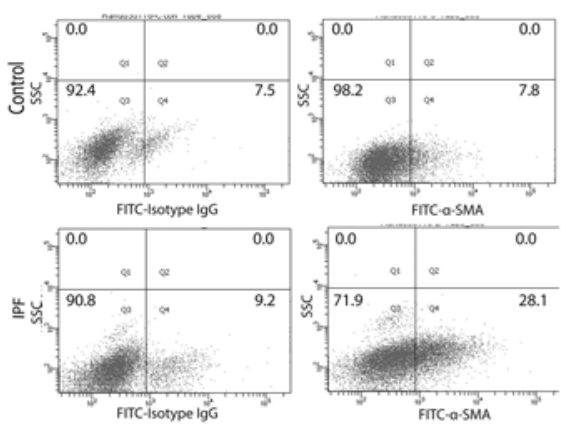

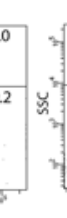

C
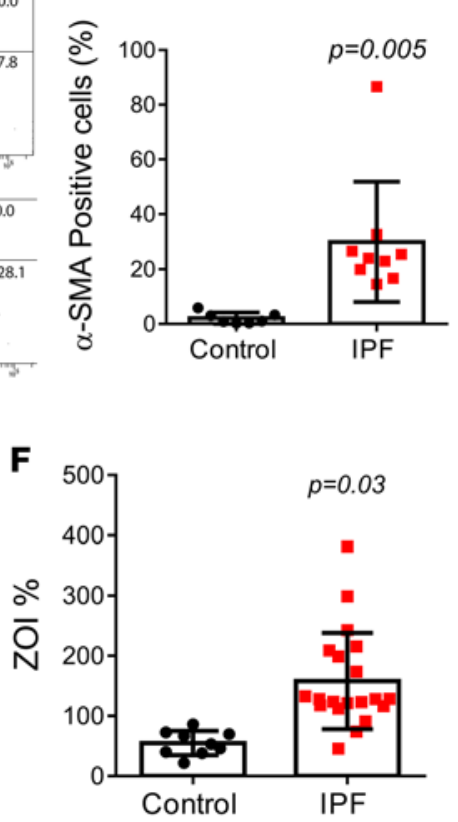

Figure 3. Quantitation of invasion in control and IPF patient pulmospheres. (A) Immunofluorescent staining for $\alpha$ smooth muscle actin ( $\alpha$-SMA) in control and IPF pulmospheres. Scale bar: $50 \mu \mathrm{m}$. (B) FACS analysis of cells of dissociated pulmospheres $(n=7)$ from 7 control subjects and 9 IPF patients. (C) $\alpha$-SMA cells were significantly increased in IPF patient pulmospheres compared with control pulmospheres. Data are expressed as mean (error bars) \pm SD. $P=0.005$, compared with control subjects, 2-tailed unpaired Student's $t$ test. Each dot represents the value for an individual subject. (D) Illustration and calculation of $3 D$ pulmosphere zone of invasion (ZOI). Schematic of pulmosphere after 12 hours of incubation in collagen gel matrix: the initial pulmosphere is delineated by a blue circle, the invading pulmosphere is delineated by dotted line covering the expanded area. (E) The formula to quantitate ZOI. (F) Differential ZOI in pulmospheres from controls $(n=9)$ and IPF patients $(n=20)$. Data are expressed as mean (error bars) \pm SD. $P=0.03$, compared with control subjects, Mann Whitney test. Each data point represents measurements of ZOI\% from 5 pulmospheres from each individual control subject and IPF patient.

antifibrotic therapy (nintedanib, $n=5$; pirfenidone, $n=4$; and N-acetylcysteine, $n=3$; total $=12$ ). We evaluated the IPF pulmosphere invasiveness in 8 subjects who were not on nintedanib or pirfenidone. There was a direct relationship between invasiveness of their lung pulmospheres as measured by basal ZOI and their clinical outcomes. Subjects with progressive disease had a high ZOI, whereas one subject who had no significant disease progression had a low ZOI (Figure 6A).

In addition, we studied the relationship between IPF lung pulmosphere ZOI and disease progression in subjects who were started on nintedanib $(n=5)$ or pirfenidone $(n=4)$. All 5 subjects treated with nintedan$\mathrm{ib}$ had nonprogressive disease by clinical criteria (Figure 6B). Of those 5 subjects with IPF, 4 had a ZOI $<1$ when their lung pulmospheres were treated with nintedanib and only one had a ZOI $>1$.

Four subjects with IPF were on therapy with pirfenidone. The pirfenidone-treated pulmospheres from 3 of those subjects had a ZOI $<1$, whereas the pulmospheres from the fourth subject had a ZOI $>1$. All subjects with pirfenidone-induced reduction in the ZOI were also clinical nonprogressors. The single subject whose pirfenidone-treated pulmospheres had a ZOI $>1$ was a clinical progressor, with an absolute drop in FVC of more than $10 \%$ in the year following diagnosis (Figure $6 \mathrm{~B}$ ).

In summary, evaluation of invasiveness as measured by the ZOI in patient pulmospheres has the potential to provide personalized evaluation of responsiveness to specific drug therapy in IPF patients. The IPF pulmospheres were tested for responsiveness, defined by a ZOI $<1$, to both nintedanib and pirfenidone. Of the 20 distinct subjects enrolled in this study, 3 patients' pulmospheres responded only to nintedanib, 4 patients' pulmospheres responded only to pirfenidone, 11 patients' pulmospheres responded to both drugs, and 2 patients' pulmospheres did not responded to either drug (Figure 6C). The individual data for patient 19 demonstrate a case in which this patient's pulmospheres responded to both drugs, whereas in patients 6 and 13, pulmospheres responded only to either nintedanib or pirfenidone, respectively. In patient 14, pulmospheres did not respond to either nintedanib or pirfenidone (Figure 6D). 
A

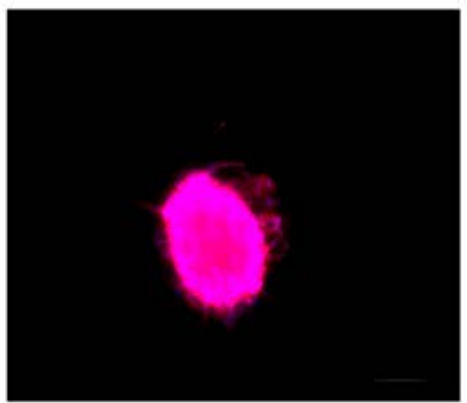

Control

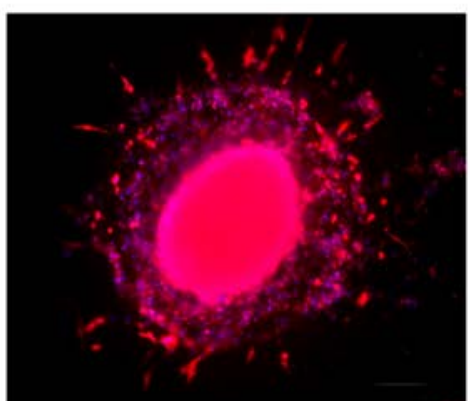

TGF- $\beta 1$

\section{c}
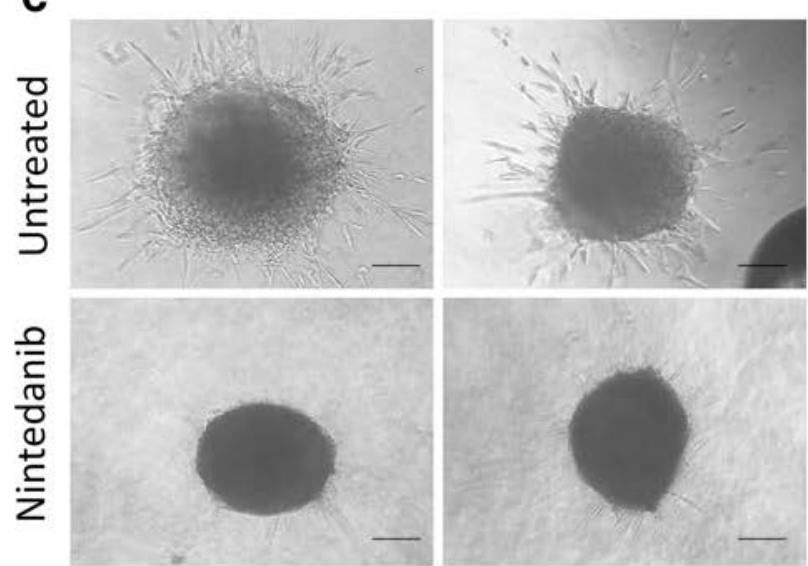

B

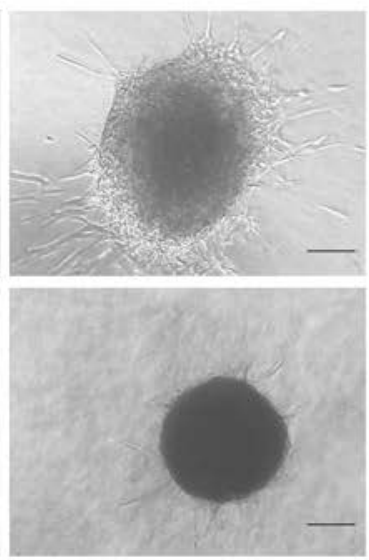

$p=0.002$

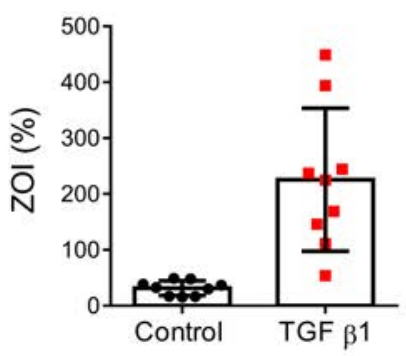

D

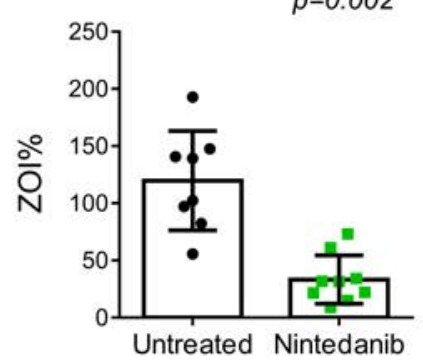

Figure 4. Modulation of invasiveness by treatment with TGF- $\beta 1$ in control pulmosphere and with nintedanib in IPF pulmosphere. (A) Representative pulmosphere from control lung not treated and treated with TCF- $\beta 1$. Scale bar: $250 \mu \mathrm{m}$. (B) Calculated zone of invasion (ZOI) from 5 lung pulmospheres from 9 controls not treated and treated with TGF- $\beta 1$. Each dot represents the mean value of calculated ZOI from 5 pulmospheres for each control subject. Data are expressed as mean (error bars) $\pm \mathrm{SD}$. $P=0.002$, compared with control pulmospheres (untreated), 2-tailed paired $t$ test. (C) Representative phase-contrast images of pulmospheres from a patient with IPF not treated and treated with nintedanib ( $1 \mu$ M) for 12 hours. Scale bar: $250 \mu \mathrm{m}$. (D) Invasiveness of pulmospheres from an individual patient not treated and treated with nintedanib. Each dot represents one pulmosphere. Data are expressed as mean (error bars) $\pm \mathrm{SD}$. $P=0.002$, compared with untreated pulmospheres, 2-tailed paired $t$ test.

\section{Discussion}

There is an urgent need to develop physiologically relevant $3 \mathrm{D}$ in vitro models for further analysis and pharmacologic research, including drug screening for IPF (18). Preclinical studies in 2D cell culture and in animal models of fibrosis fail to replicate the dynamic and progressive disease or the microenvironment in the lungs of patients with IPF. It is well recognized that multiple cell types and pathways play important roles in the development of IPF (19). The altered ECM and immune microenvironment surrounding the invading myofibroblasts and other cell types contribute to the anatomic and physiological changes representing the lung the IPF $(20,21)$.

In our study, we used lung cells from the lung digest single-cell suspension (passage limited to 0 or 1 ) in an attempt to recapitulate the patient lung ex vivo. Growing cells through several passages alters cellular phenotype. Others have developed lung 3D culture systems from IPF lung biopsies $(22,23)$. However, because their goals in examining 3D cultures were different, they used other techniques to develop them. Henry et al. used lung tissue explants with an outgrowth of cells occurring for approximately 1 week in an attempt to allow lung stem cells to proliferate. The aim of the study was to regenerate stem cells for the treatment of IPF, not to provide a tool for personalized precision therapy for unique individual patients, which is the goal of the present study (22). Another method, that of Wilkinson et al. used multiple alginate beads as a scaffold to grow multicellular structures on the surface of the beads using STEMCCA Cre-Excisable Constitutive Polycistronic Lentivirus-transduced cells to reprogram them as pluripotent cells (23). This method of preparation does not recapitulate the physical or the cell-autonomous characteristic of an individual patient lung.

In our present study, we developed pulmospheres from lung tissue obtained via video-assisted thoracic surgery biopsy of IPF subjects and from failed donor lung explants as normal controls. A recent study of patients with fibrotic lung disease used bronchoscopic lung cryobiopsy in over 117 patients 
A Fold change in ZOI after drug treatment $=\frac{\text { ZOI\% with drug treatment }}{\text { ZOI\% without drug treatment }}$

B
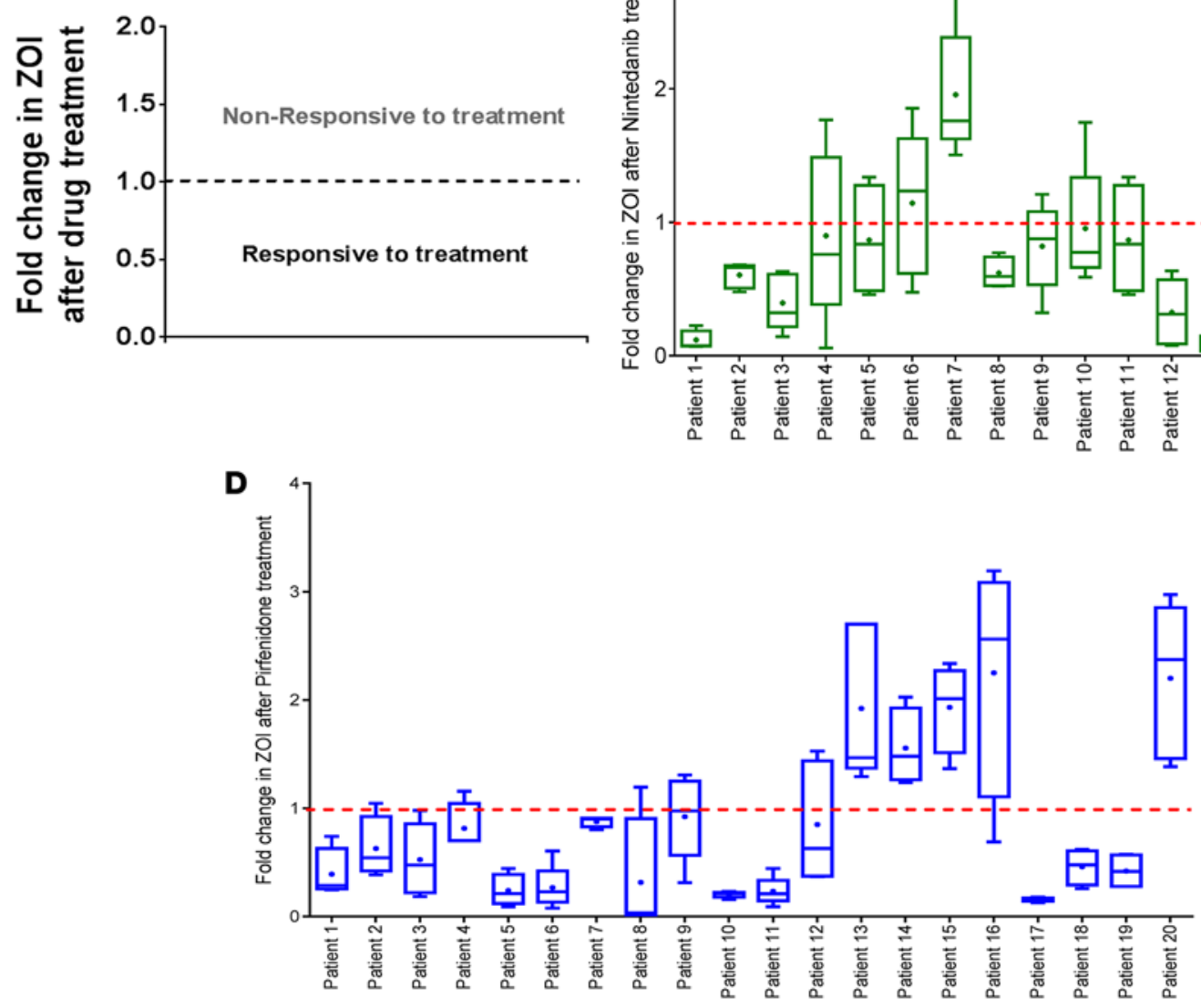

Figure 5. Differential invasiveness of pulmospheres from patients with IPF following exposure to nintedanib and pirfenidone. (A) Measurement of fold change of zone of invasion (ZOI) was calculated as the ratio of ZOI\% with treatment to ZOI\% without treatment in vitro with antifibrotic drug. (B) Median values of ZOI $>1$ represent pulmospheres nonresponsive to the treatment, and median values of ZOI $<1$ represent pulmospheres responsive to drug treatment. For each patient tested, 5 pulmospheres were seeded without either test drug; 5 pulmospheres were seeded with nintedanib ( $1 \mu \mathrm{M})$; and 5 pulmospheres were seeded with pirfenidone $(1 \mathrm{mM})$. ZOI fold change values were obtained from the ratio of ZOI\% with treatment to ZOI\% without treatment of pulmospheres in vitro with antifibrotic drug for each patient. Box-and-whisker plots for IPF patient pulmospheres treated with (C) nintedanib and (D) pirfenidone. Whiskers show maximum to minimum values. Lines in boxes represent median values. The "'+"signs inside boxes represent the mean values.

and was found to provide useful information in the analysis of patient subtype $(24,25)$. It is unclear if pulmospheres can be obtained from lung cryobiopsies, but given that each pulmosphere requires only $8-10,000$ cells, it is very possible that it could be an attractive alternative to obtaining tissue via video-assisted thoracic surgery $(24,25)$. The normal control lung tissues were obtained from failed lung transplant donors. We found that lung pulmospheres formed rapidly, within 12 hours of culture on nonadherent 96-well plates, and contained multiple lung cell types, including CD11b-, SPC-, and CD31-positive cells. The majority of the cells from the lungs of patients with IPF were $\alpha$-SMA-positive cells. There was a marked difference in the expression of ECM proteins in the pulmospheres from patients with IPF compared with controls. Interestingly both $\alpha$-SMA expression and FSP1 expression were markedly different in $2 \mathrm{D}$ culture as compared with $3 \mathrm{D}$ culture, suggesting that $3 \mathrm{D}$ culture removes some of the artificial changes induced by culturing cells on hard surfaces. The conventional 2D culture exaggerates the in vivo expression of SMA and FSP1. Conventional 2D culturing methods on plastic provide stiff matrices for cell growth. The stiff matrices provide a high-tensile state that leads to cytoskeletal reorganization and the formation of stress fibers (11). Pulmospheres mimic in vivo conditions with softer matrices and cell-cell interaction.

Invasion of myofibroblasts in IPF is distinct from cellular migration (6). The ability of fibroblasts to 
A Patients not on nintedanib or pirfenidone

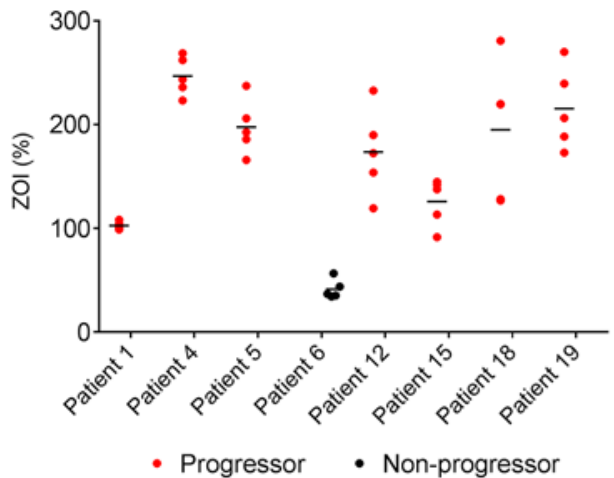

C

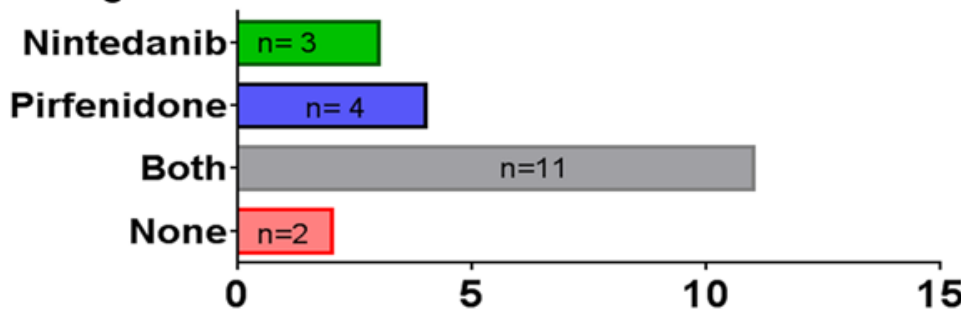

B Patients on nintedanib or pirfenidone

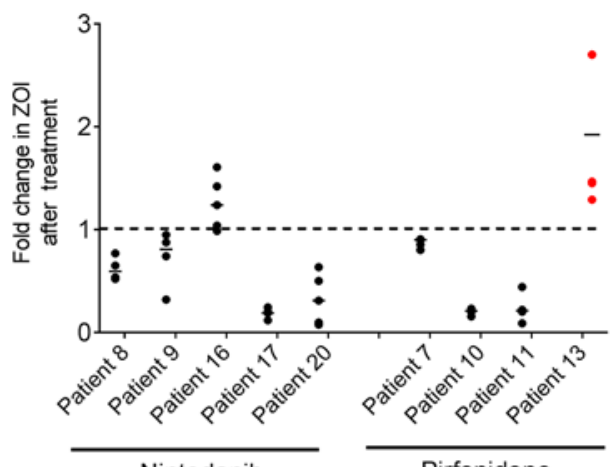

Nintedanib

Pirfenidone

\section{Number of IPF subjects whose pulmosphere respond to nintedanib and/or pirfenidone}

D

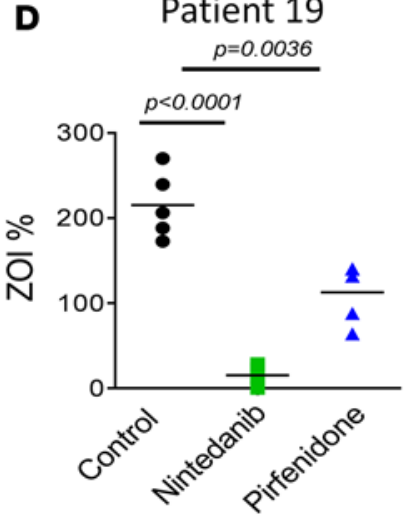

Patient 13

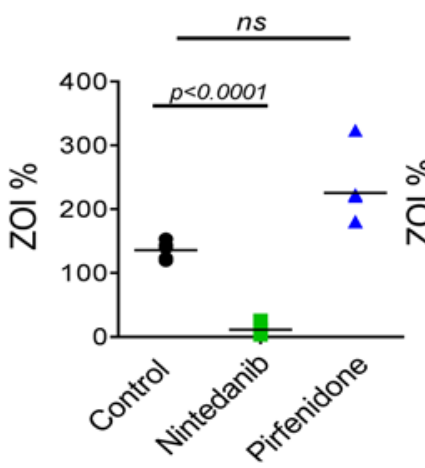

Patient 6

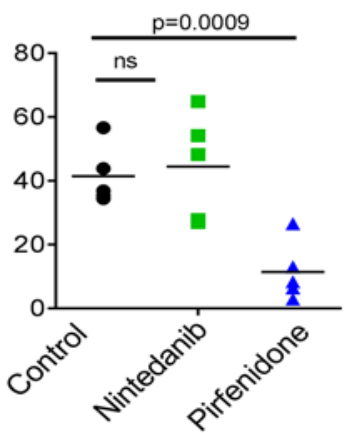

Patient 14

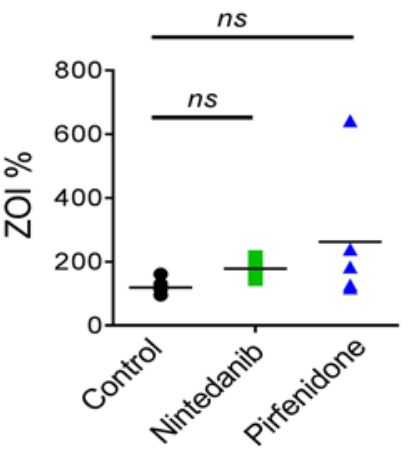

Figure 6. Correlation between pulmosphere invasiveness and disease progression in patients with IPF. (A) Relationship between clinical outcomes in individual patients and invasiveness of patient pulmosphere. These 8 patients with IPF were not on any medication directed to IPF. Progressors were defined as those patients with a drop in forced vital capacity (FVC) $>10 \%$ or a drop in $\mathrm{DL}_{c 0}>15 \%$ in 12 months or death. (B) Relationship between patient disease progression and pulmosphere invasiveness in response to nintedanib and pirfenidone. Five patients were on nintedanib, and four patients were on pirfenidone (red represents progressors and black represents nonprogressors. Median values of ZOI $>1$ represent pulmospheres nonresponsive to the treatment, and median values of $\mathrm{ZOI}<1$ represent pulmospheres responsive to drug treatment. (C) Number of patients whose pulmospheres were responsive to nintedanib only (green), pirfenidone only (blue), either nintedanib or pirfenidone (gray), or neither (red). (D) Individual patient responses to nintedanib and pirfenidone. Patient 19 responded to both nintedanib and pirfenidone, with greater inhibition of invasiveness of pulmospheres with nintedanib than pirfenidone. Patient 13 responded only to nintedanib. Patient 6 responded only to pirfenidone. Patient 14 did not respond to nintedanib or pirfenidone. All $P$ values, compared with untreated pulmospheres, 2-tailed paired $t$ test. Data were obtained from 5 pulmosphere for each group (untreated, nintedanib treated, and pirfenidone treated) per patient. "Response" is defined as a decrease in the ZOI\% of each patient's pulmosphere after treatment with nintedanib or pirfenidone as compared with untreated control.

invade has been recently recognized in $\operatorname{IPF}(13,26)$. Lamellipodia protrude from the leading edge of normally migrating cells. Podosomes or invadopodia lead invading fibroblasts with activation of cell surface proteases and remodeling of the $\operatorname{ECM}(12,27,28)$. A recent study from our group demonstrated that mechanosensing of stiffened matrix by $\beta_{6}$ integrins confers an invasive phenotype of IPF lung myofibroblasts and mediates experimental lung fibrosis (29). IPF is also characterized by basement membrane destruction, which allows fibroblasts to invade into the provisional matrices that develop in alveoli after injury. This 
cell-autonomous invasive quality in the absence of exogenous mediators of fibroblasts and myofibroblasts from patients with IPF is markedly different from the non-cell-autonomous invasion of pulmospheres from control patient lungs, which requires exogenous stimulation, such as TGF- $\beta 1$, to elicit the invasive phenotype $(9,13)$. There is emerging recognition of the heterogeneity of IPF pathogenesis, including cell-autonomous behavior and chemoinvasive mediators in the local microenvironment (6). This suggests that each individual patient with IPF has numerous factors that contribute to the phenotype of invasive myofibroblasts (30). The variability in the invasiveness of pulmospheres from control patients and IPF is clearly demonstrated in Figure 3F. There are also remarkable differences in the invasiveness of pulmospheres from individual patients with IPF. These data lend credence to the concept that the individual response of a particular patient's pulmosphere is a reflection of the multitude of coalescing factors that influence invasion. Invasion is a biological phenomenon quantitatively and qualitatively specific to individual patient endotype and reflects their disease. We utilized this phenomenon to identify personalized responses of individual patient pulmospheres to the antifibrotic drugs, nintedanib $(3,31)$ and pirfenidone $(2,7)$.

Both nintedanib and pirfenidone have been shown to slow down the progression of pulmonary fibrosis in patients with IPF. However, the timing of initiation of therapy and choice of antifibrotic drug remain a subject of controversy $(32,33)$. It is unclear if patients will respond to either drug, nintedanib or pirfenidone, given in combination or serially. The mechanism of action of pirfenidone is thought to be through inhibition of TGF- $\beta 1$ (34), with subsequent downregulation of inflammatory cytokines and oxidative stress (1). Nintedanib is thought to inhibit tyrosine kinases and has been more recently recognized to induce noncanonical autophagy in fibroblasts, while also inhibiting TGF- $\beta 1$ signaling $(35,36)$. There are at least 10 ongoing clinical trials targeting various mediators with calls for using a multitargeted approach during the course of the disease (36).

Longitudinal changes in FVC or diffusion capacity for carbon monoxide $\left(\mathrm{DL}_{\mathrm{CO}}\right)$ are predictive of prognosis in IPF as compared with baseline values. A drop of $10 \%$ or greater decline in FVC is associated with a more than 2-fold increase in the risk of mortality $(37,38)$. We defined subjects with these parameters as "clinical progressors," and those without were defined as "clinical nonprogressors." IPF subjects on nintedanib or pirfenidone who were clinical progressors had lung pulmospheres that reflected their clinical response. However, there was individual variability in the response. Some subjects who were on nintedanib had a lower ZOI in their pirfenidone-treated pulmospheres and vice versa. There were also subjects whose pulmosphere ZOI continued to increase despite treatment with either drug.

Other investigators have used lung cells to develop 3D cultures of invading fibroblasts or stem cells obtained from lung tissue from human or mice lung $(22,39)$. Henry et al. used lung tissue pieces in culture to recoup "outgrowth cells" to form lung pulmospheres, which were found to be enriched for stem cells and protected from bleomycin lung injury in mice (22). A group from Germany performed a pathway analysis on the invading fibroblasts, which has provided important information on the signaling pathways, which mediate the process of invasion in lung fibroblasts (39). A key difference in the pulmospheres obtained in our studies is that they were grown immediately following resection of lung tissue and were developed within 24 hours from lung cell suspensions and adherent cells following the initial passage through filter and removal of red blood cells. No attempt was made to enrich for specific cell types. This allowed us to test for drug responsiveness via analysis of invasion immediately following the development of pulmospheres, with final results obtained at 48 hours following tissue procurement. The rapidity with which it is possible to specify which drug causes the greatest inhibition of pulmosphere invasion is an attractive aspect of this study. Importantly pulmospheres lend themselves not only to evaluating specific targeted drug therapy for individual patients, but also to high-throughput screening for drugs that are still in the pipeline.

There are several caveats to our study. Pulmospheres were obtained from lung biopsies via video-assisted thoracic surgery. It is important to find less invasive methods to obtain tissue in an era in which the diagnosis of IPF can be made on CT scans. It is possible that bronchoscopic cryobiopsies may be a useful method for obtaining tissue to form pulmospheres. Though every attempt was made to initiate the formation of pulmospheres with the same number of cells, pulmospheres in different patients developed into different sizes. It is possible that the baseline size of the pulmosphere prior to incubation with drug is dependent on the rate of growth of cells and/or the formation of ECM inside the pulmosphere. However, the starting size of the pulmosphere was taken into account in the analysis of invasiveness to remove it as a confounding factor. Though a total of 29 subjects were studied, only 20 of the patients had IPF, and smaller numbers were already on therapy with pirfenidone ( 4 patients) or nintedanib ( 5 patients). The goal in the development of lung pulmospheres from patients with IPF was to imitate the lung microenvironment with 
multiple cell types and ECM components of individual patients. They represent the local tissue that was obtained in the biopsy. Given that the disease is anatomically patchy, with fibrosis unequally spread across lobes and locations in the lung, our biopsy tissue may not reflect the totality of disease in the lung.

This study is important because for the first time to our knowledge an attempt was made to develop a global representation of lung tissue from patients with IPF using 3D cultures to better understand their individual response to specific therapy, without focusing on a specific cell type or signaling pathway. It was recognized that pulmospheres have a multitude of cell types and mediators that regulate the biological phenomenon of invasion that characterizes IPF. This allows us to test a variety of therapeutic modalities with the goal to personalize therapy and develop new targets for a disease whose mortality remains unacceptably high.

\section{Methods}

Patient recruitment and clinical data. We obtained lung tissue from 20 consecutive subjects who were evaluated in the University of Alabama at Birmingham Interstitial Lung Disease Program and required surgical lung biopsy for the diagnosis of IPF and from explanted IPF lungs over a period of 3 years. Control, normal lung tissues were obtained from 9 failed lung transplant donors. The final diagnosis of IPF was established by a multidisciplinary discussion group following 2011 ATS/ERS guidelines (40). As part of usual clinical care, all subjects underwent pulmonary function tests that included FVC and $\mathrm{DL}_{\mathrm{CO}}$ during both their initial and follow-up visits.

Longitudinal changes in FVC or $\mathrm{DL}_{\mathrm{CO}}$ are suggested to be predictive of outcomes in IPF. An absolute drop of $10 \%$ or greater in FVC in a 6-month period is associated with a more than 2 -fold increase in the risk of mortality $(37,38,41)$. Hence, in this study, subjects who died or dropped their $\mathrm{FVC}_{\text {or }} \mathrm{DL}_{\mathrm{CO}}$ in the first year after diagnosis by more than $10 \%$ and $15 \%$, respectively, were classified as "progressors." Otherwise, they were classified as "nonprogressors."

Lung tissue and single-cell suspension preparation. Lung tissue samples from patients or controls were washed with cold PBS solution. For approximately $6 \mathrm{~mm} \times 6 \mathrm{~mm}$ tissue each, $5 \mathrm{ml}$ of collagenase A was incubated with the tissue in a $50-\mathrm{ml}$ tube for 30 minutes in a $37^{\circ} \mathrm{C}$ water bath. The tube was gently agitated for a few seconds every 10 minutes during this incubation. PBS $(20 \mathrm{ml})$ was added to the tube after 30 minutes of incubation. The tube was then vigorously shaken for 30 seconds to disassociate the lung tissue, and the resulting single-cell suspension was filtered through a $100-\mu \mathrm{m}$ filter. The filtered single-cell suspension was verified by light microscopy and was centrifuged for 5 minutes at $300 \mathrm{~g}$. The cell pellet was washed with complete DMEM (consisting of $10 \% \mathrm{FCS}$, penicillin and streptomycin, and Glutamax, Invitrogen) and resuspended in $5 \mathrm{ml}$ of ACK lysis buffer (catalog A1049201, Gibco) to lyse red blood cells. Tubes were incubated at room temperature for 5 minutes with occasional shaking. The reaction was stopped by diluting the ACK lysis buffer with $30 \mathrm{ml}$ of PBS. Cells were centrifuged at $300 \mathrm{~g}$ at room temperature for 5 minutes. The supernatant was removed carefully, and the pellet was resuspended in complete growth media. Cells were plated in a T-25 flask. These passage $0-1$ cells were used for preparation of 3D spheroids.

Preparation of poly-HEMA-coated plates. Stock solution of poly-HEMA (120 mg/ml) was prepared in $95 \%$ ethanol. Working solution of poly-HEMA was obtained at final concentration of $5 \mathrm{mg} / \mathrm{ml}$ using $95 \%$ ethanol. The working solution was vortexed briefly for 30 seconds. A total $160-\mu 1$ volume of working solution was added per well in 96-well U-bottom plates. Plates were left at room temperature in sterile laminar hood for 24 hours (until the solution is evaporated). These coated plates were used for preparation of pulmospheres.

Preparation of pulmospheres. Primary lung cells were detached with trypsin and neutralized with $5 \mathrm{ml}$ growth media. Cells were collected by centrifugation at $300 \mathrm{~g}$ for 5 minutes at room temperature. The total cell pellet was resuspended in $2 \mathrm{ml}$ complete DMEM medium. A portion of the cells was stained with trypan blue and counted to check for cell density and viability. 8,000-10,000 cells (depending upon total number of cells and assays) were added per well in poly-HEMA-coated plate. The plate was centrifuged (IEC-Centra 7R) at $200 \mathrm{~g}$ at room temperature for 1 minutes. Plates were incubated for 16 hours at $37^{\circ} \mathrm{C}$ in $\mathrm{CO}_{2}(5 \%)$ incubator.

The resulting 3D spheroids were termed "pulmospheres." We defined pulmospheres as in vitro 3D clusters of cells or spheroids derived exclusively from primary lung tissue and inclusive of lung cell types reflective of those in situ, in the patient.

Fluorescent automated cell sorting. Pulmospheres were disaggregated with trypsin $(0.025 \%) /$ EDTA and washed with FACS buffer (5\% FCS, 0.03\% sodium azide-PBS).

For CD11b and CD31 staining, living cells were incubated for 30 minutes at $4^{\circ} \mathrm{C}$ with primary anti- 
body conjugated with PE (anti-CD11b antibody, catalog 101207, and anti-CD31 antibody, catalog 303106, BioLegend) and washed with FACS buffer. For SPC (catalog ab32575, Abcam), staining cells were incubated for 30 minutes at $4^{\circ} \mathrm{C}$ and eventually washed with FACS buffer. Cells were incubated for 30 minutes at $4^{\circ} \mathrm{C}$ with primary antibody conjugated and followed by anti-mouse AlexaFluor 647 (Molecular Probes, Life Technologies) secondary antibody for 30 minutes at $4^{\circ} \mathrm{C}$.

$\alpha$-SMA (catalog ab32575, Abcam) staining was performed after cell fixation and permeabilization using the Intrastain kit (DAKO) according to the instructions of the manufacturer. Cells were incubated for 30 minutes at $4^{\circ} \mathrm{C}$ with primary antibody conjugated and followed by anti-mouse AlexaFluor 488 secondary antibody for 30 minutes at $4^{\circ} \mathrm{C}$.

Labeled cells were analyzed by FACS, and data were computed using software (BD CellQuest). Gating was performed on the basis of negative control staining profiles, obtained by substituting primary antibodies with isotypic nonimmune IgGs.

Immunohistochemistry of pulmospheres. Pulmospheres were washed and fixed in formalin for 24 hours at room temperature. Formalin was pipetted out, and $100 \mu 1$ of warmed (at $80^{\circ} \mathrm{C}$ ) Histogel (Richard Allen Scientific, catalog HG-4000-012) was added to the pulmospheres. The Histogel-pulmosphere suspension was refrigerated until the gel became firm. The gel was taken out from the tube with the help of a spatula and kept in a cassette. The cassette was incubated in formalin solution overnight. These cassettes were processed for paraffin sectioning to obtain 4-micron thin sections.

Immunofluorescent staining of pulmospheres. After blocking in PBST (0.1\% Triton X-100 in PBS) containing 3\% Bovine Serum Albumin (Sigma-Aldrich) overnight at $4{ }^{\circ} \mathrm{C}$ and washing in PBST (twice for 15 minutes), pulmospheres were incubated with primary antibodies diluted in PBST on a gently rocking rotator at $4^{\circ} \mathrm{C}$ for 48 hours and rinsed in PBST (4 times for 30 minutes). When necessary, pulmospheres were then incubated in appropriate AlexaFluor-conjugated secondary antibodies (Molecular Probes, Life technologies) for 24 hours. Cell nuclei were counterstained by DAPI (Invitrogen) diluted 1:500 in PBS for 40 minutes at room temperature. Immunolabeled pulmospheres were imaged using confocal microscopy.

For paraffin sections, immunofluorescent staining was performed as described previously (42). The primary antibodies used for staining were Fibronectin-EDA antibody (catalog sc-59826, Santa Cruz Inc.), SPC (catalog ab40879, Abcam), CD11b (catalog ab8878, Abcam), CD31 (catalog MA 3105, Thermo Scientific), $\alpha$-SMA (catalog ab32575, Abcam), Collagen type IV (catalog ab6586, Abcam), and Collagen type I (catalog 600-401-103-0.5, Rockland Inc.). All immunofluorescence stained slides were costained with DAPI for visualization of the nucleus.

Protein lysate preparation and Western blotting. The growth medium was aspirated and pulmospheres were washed with PBS. $25 \mu$ RIPA lysis buffer (Sigma-Aldrich) with phosphatase inhibitor cocktail (Novus Biologicals) was stored at $-80^{\circ} \mathrm{C}$. Samples underwent 4 freeze-thaw cycles to solubilize pulmospheres in RIPA lysis buffer (Gibco). The pulmosphere lysate was pipetted gently (avoiding froth) and used for protein estimation by the MicroBCA protein assay kit (Pierce). Lysates were prepared and Western blotting was performed as described elsewhere (38). Primary antibodies $\alpha$-SMA (1:1,000, sc-130616, Santa Cruz Inc.), FSP1 (dilution 1:800, 07-2274 clone S100A4, Millipore), and $\beta$-actin (dilution 1:2,000, Sigma-Aldrich) were used in 5\% milk-TBST solution.

Analysis of invasion by pulmospheres. Pulmospheres were formed following 16 hours of incubation of seeded cells in poly-HEMA-coated plates. Analysis of invasion by pulmospheres was initiated immediately after the 16-hour incubation. Collagen solution was prepared by mixing precooled (at $\left.4^{\circ} \mathrm{C}\right) 2.1 \mathrm{ml}$ of collagen type I solution $(3.98 \mathrm{mg} / \mathrm{ml}$ ) (Corning) and $6.2 \mathrm{ml}$ of DMEM (Invitrogen). $\mathrm{NaOH}(1 \mathrm{M})$ was used to set $\mathrm{pH}$ 7-7.4 of this collagen-DMEM solution. The solution was mixed gently by pipetting, avoiding the introduction of air bubbles. Of this solution, $50 \mu \mathrm{l} /$ well was added to cover the bottom of flat-bottom 96 -well plates. This bottom layer prevents contact of pulmosphere cells with the plastic of the well. We let the collagen set on a flat surface at $37^{\circ} \mathrm{C}$ in a $\mathrm{CO}_{2}$ incubator for at least 2 hours. Growth medium was removed from pulmospheres culture plate. Single pulmospheres were seeded onto the collagen-coated well with $100 \mu 1$ of the collagen-DMEM solution with nintedanib $(1 \mu \mathrm{M})$ or pirfenidone $(1 \mathrm{mM})$ or media alone without test drug in a single well. In total, 5 pulmospheres were seeded without any test drug in collagen-DMEM solution into 5 individual wells; 5 pulmosphere were seeded with nintedanib-containing collagen-DMEM solution into 5 individual wells; and 5 pulmospheres were seeded with pirfenidone-containing collagen-DMEM solution into 5 individual wells for each patient tested. These plates were incubated for 30 minutes at $37^{\circ} \mathrm{C}_{\text {in }} \mathrm{CO}_{2}$ incubator. $50 \mu \mathrm{DMEM}$ (with nintedanib $[1 \mu \mathrm{M}]$ or pirfenidone [1 $\mathrm{mM}]$ or without test drugs) was added 
to each well. Plates were incubated at $37^{\circ} \mathrm{C}$ in a $\mathrm{CO}_{2}$ incubator overnight. Images were acquired with a Carl Zeiss AxioCam color camera (Carl Zeiss Vision $\mathrm{GmbH})$ and analyzed using AxioVision LE Imaging System software (Carl Zeiss Vision $\mathrm{GmbH}$ ).

In the AxioVision LE Imaging System software, the area covered by the invading cells was selected and measured $(\mathrm{H})$. The center mass of the pulmosphere was also outlined and measured $(\mathrm{R})$. The percentage of total invaded area $(\mathrm{ZOI} \%)$ was calculated by $(\mathrm{H}-\mathrm{R}) / \mathrm{R} \times 100$. Percentage invasion area was calculated. Measurement of fold change of ZOI was calculated as the ratio of ZOI\% with treatment to ZOI\% without treatment in vitro with antifibrotic drug.

Statistics. The results are presented as the mean $\pm \mathrm{SD}$, unless specified otherwise. Comparisons between any two groups were performed using 2-tailed unpaired Student's $t$ tests and 2-tailed paired $t$ tests. The comparisons within group were performed using paired $t$ test. Response rate to each treatment was calculated. An exact $95 \%$ confidence interval around the response rate was calculated using the Clopper-Pearson method. Differences were considered statistically significant at $P<0.05$.

Study approval. The University of Alabama at Birmingham Institutional Review Board reviewed and approved this project (UAB-IRB F130429005). Written informed consent was obtained from participants.

\section{Author contributions}

RS conducted experiments, acquired data, analyzed data, and wrote the manuscript. FJL, ZW, and HL conducted processing of lung samples and experiments. GL, YZ, TL, RL, and SR discussed the results and implications and commented on the manuscript. SB conducted statistical analysis in the study. JA provided biopsy samples, patient clinical outcome data, discussed results and their implications, and edited the manuscript. VJT gave conceptual advice and edited the manuscript. VBA conceived and designed the study, discussed results and their implications, and wrote the manuscript.

\section{Acknowledgments}

This work was supported by NIH grant P01 HL114470 (to VJT and VBA).

Address correspondence to: Veena B. Antony, 1530 3rd Avenue So, THT-422, Birmingham, Alabama 35294, USA. Phone: 205.975.3255; E-mail: vantony@uabmc.edu.

1. Maher TM. Pirfenidone in idiopathic pulmonary fibrosis. Drugs Today. 2010;46(7):473-482.

2. King TE, et al. A phase 3 trial of pirfenidone in patients with idiopathic pulmonary fibrosis. NEngl J Med. 2014;370(22):2083-2092.

3. Richeldi L, et al. Efficacy and safety of nintedanib in idiopathic pulmonary fibrosis. N Engl J Med. 2014;370(22):2071-2082.

4. Kolb M, Collard HR. Staging of idiopathic pulmonary fibrosis: past, present and future. Eur Respir Rev. 2014;23(132):220-224

5. Saini G, et al. $\alpha v \beta 6$ integrin may be a potential prognostic biomarker in interstitial lung disease. Eur Respir J. 2015;46(2):486-494.

6. Ahluwalia N, et al. Fibrogenic lung injury induces non-cell-autonomous fibroblast invasion. Am J Respir Cell Mol Biol. 2016;54(6):831-842.

7. Noble PW, et al. Pirfenidone in patients with idiopathic pulmonary fibrosis (CAPACITY): two randomised trials. Lancet. 2011;377(9779):1760-1769.

8. Lancaster MA, Knoblich JA. Organogenesis in a dish: modeling development and disease using organoid technologies. Science. 2014;345(6194):1247125.

9. Burgstaller G, Oehrle B, Koch I, Lindner M, Eickelberg O. Multiplex profiling of cellular invasion in 3D cell culture models. PLoS One. 2013;8(5):e63121.

10. Xu JY, et al. Generation of induced cardiospheres via reprogramming of skin fibroblasts for myocardial regeneration. Stem Cells 2016;34(11): 2693-2706.

11. Zhou Y, et al. Inhibition of mechanosensitive signaling in myofibroblasts ameliorates experimental pulmonary fibrosis. $J$ Clin Invest. 2013;123(3):1096-1108

12. Li Y, et al. Severe lung fibrosis requires an invasive fibroblast phenotype regulated by hyaluronan and CD44. J Exp Med. 2011;208(7):1459-1471.

13. Karvonen HM, et al. Myofibroblasts in interstitial lung diseases show diverse electron microscopic and invasive features. Lab Invest. 2012;92(9):1270-1284.

14. Hecker L, et al. Reversal of persistent fibrosis in aging by targeting Nox4-Nrf2 redox imbalance. Sci Transl Med. 2014;6(231):231ra47.

15. Hostettler K, Zhong J, Tamm M, Lardinois D, Roth M. Effect of pirfenidone on TGF- $\beta$-induced pro-fibrotic effects in primary human lung cells derived from patients with idiopathic pulmonary fibrosis. Eur Respir J. 2014;44 (Supp 58): P763.

16. Hostettler KE, et al. Anti-fibrotic effects of nintedanib in lung fibroblasts derived from patients with idiopathic pulmonary fibrosis. Respir Res. 2014;15:157.

17. Lehtonen ST, et al. Pirfenidone and nintedanib modulate properties of fibroblasts and myofibroblasts in idiopathic pulmonary fibrosis. Respir Res. 2016;17:14. 
18. Picollet-D'hahan N, et al. A 3D toolbox to enhance physiological relevance of human tissue models. Trends Biotechnol. 2016;34(9):757-769

19. Thannickal VJ, Zhou Y, Gaggar A, Duncan SR. Fibrosis: ultimate and proximate causes. J Clin Invest. 2014;124(11):4673-4677.

20. Kulkarni T, de Andrade J, Zhou Y, Luckhardt T, Thannickal VJ. Alveolar epithelial disintegrity in pulmonary fibrosis. Am J Physiol Lung Cell Mol Physiol. 2016;311(2):L185-L191.

21. Ding Q, et al. New insights into the pathogenesis and treatment of idiopathic pulmonary fibrosis. Drugs. 2011;71(8):981-1001

22. Henry E, et al. Adult lung spheroid cells contain progenitor cells and mediate regeneration in rodents with bleomycin-induced pulmonary fibrosis. Stem Cells Transl Med. 2015;4(11):1265-1274.

23. Wilkinson DC, et al. Development of a three-dimensional bioengineering technology to generate lung tissue for personalized disease modeling. Stem Cells Transl Med. 2016;5(1):1-12.

24. Ravaglia C, et al. Safety and Diagnostic Yield of Transbronchial Lung Cryobiopsy in Diffuse Parenchymal Lung Diseases: A Comparative Study versus Video-Assisted Thoracoscopic Lung Biopsy and a Systematic Review of the Literature. Respiration. 2016;91(3):215-227.

25. Tomassetti S, et al. Bronchoscopic Lung Cryobiopsy Increases Diagnostic Confidence in the Multidisciplinary Diagnosis of Idiopathic Pulmonary Fibrosis. Am J Respir Crit Care Med. 2016;193(7):745-752.

26. White ES, et al. Integrin alpha4beta1 regulates migration across basement membranes by lung fibroblasts: a role for phosphatase and tensin homologue deleted on chromosome 10. Am J Respir Crit Care Med. 2003;168(4):436-442.

27. Wolf K, et al. Collagen-based cell migration models in vitro and in vivo. Semin Cell Dev Biol. 2009;20(8):931-941.

28. Lovgren AK, et al. $\beta$-arrestin deficiency protects against pulmonary fibrosis in mice and prevents fibroblast invasion of extracellular matrix. Sci Transl Med. 2011;3(74):74ra23.

29. Chen $\mathrm{H}$, et al. Mechanosensing by the $\alpha 6$-integrin confers an invasive fibroblast phenotype and mediates lung fibrosis. Nat Commun. 2016;7:12564.

30. Ding Q, et al. FAK-related nonkinase is a multifunctional negative regulator of pulmonary fibrosis. Am J Pathol. 2013;182(5):1572-1584.

31. Richeldi L, et al. Design of the INPULSIS ${ }^{\mathrm{TM}}$ trials: two phase 3 trials of nintedanib in patients with idiopathic pulmonary fibrosis. Respir Med. 2014;108(7):1023-1030.

32. King CS, Nathan SD. POINT: Should All Patients With Idiopathic Pulmonary Fibrosis, Even Those With More Than Moderate Impairment, Be Treated With Nintedanib or Pirfenidone? Yes. Chest. 2016;150(2):273-275.

33. Brown KK. COUNTERPOINT: Should All Patients With Idiopathic Pulmonary Fibrosis, Even Those With More Than Moderate Impairment, Be Treated With Nintedanib or Pirfenidone? No. Chest. 2016;150(2):276-278.

34. Shihab FS, Bennett WM, Yi H, Andoh TF. Pirfenidone treatment decreases transforming growth factor-beta1 and matrix proteins and ameliorates fibrosis in chronic cyclosporine nephrotoxicity. Am J Transplant. 2002;2(2):111-119.

35. Chaudhary NI, et al. Inhibition of PDGF, VEGF and FGF signalling attenuates fibrosis. Eur Respir J. 2007;29(5):976-985.

36. Rangarajan S, et al. Novel mechanisms for the antifibrotic action of nintedanib. Am J Respir Cell Mol Biol. 2016;54(1):51-59.

37. du Bois RM, et al. Forced vital capacity in patients with idiopathic pulmonary fibrosis: test properties and minimal clinically important difference. Am J Respir Crit Care Med. 2011;184(12):1382-1389.

38. Richeldi L, et al. Relative versus absolute change in forced vital capacity in idiopathic pulmonary fibrosis. Thorax. 2012;67(5):407-411.

39. Oehrle B, et al. Validated prediction of pro-invasive growth factors using a transcriptome-wide invasion signature derived from a complex 3D invasion assay. Sci Rep. 2015;5:12673.

40. Raghu G, et al. An Official ATS/ERS/JRS/ALAT Clinical Practice Guideline: Treatment of Idiopathic Pulmonary Fibrosis. An Update of the 2011 Clinical Practice Guideline. Am J Respir Crit Care Med. 2015;192(2):e3-19.

41. Hunninghake GM. A new hope for idiopathic pulmonary fibrosis. N Engl J Med. 2014;370(22):2142-2143.

42. Surolia R, et al. Heme oxygenase-1-mediated autophagy protects against pulmonary endothelial cell death and development of emphysema in cadmium-treated mice. Am J Physiol Lung Cell Mol Physiol. 2015;309(3):L280-L292. 